\title{
Integrated diagnostic approach of pediatric neuromuscular disorders
}

Ha Neul Lee and Young-Mock Lee*

Department of Pediatrics, Yonsei University College of Medicine, Seoul, Korea

Clinical and genetic heterogeneity in association with overlapping spectrum is characteristic in pediatric neuromuscular disorders, which makes confirmative diagnosis difficult and time consuming. Considering evolution of molecular genetic diagnosis and resultant upcoming genetically modifiable therapeutic options, rapid and cost-effective genetic testing should be applied in conjunction with existing diagnostic methods of clinical examinations, laboratory tests, electrophysiologic studies and pathologic studies. Earlier correct diagnosis would enable better clinical management for these patients in addition to new genetic drug options and genetic counseling.

Key words: Pediatrics, Neuromuscular diseases, Phenotype, Molecular diagnostic techniques.

\section{Introduction}

Pediatric neuromuscular disorders consist of different diseases originating from abnormality in peripheral nervous system, neuromuscular junction and skeletal muscle. Typically, clinical symptoms and signs are nonspecific and overlapping. Confirming diagnosis is not easy due to this heterogeneity, which also applies for the genetic perspective. Conventional diagnostic approach included stepwise evaluation of laboratory study, electrophysiologic study such as electromyography (EMG) and nerve conduction study (NCS) in combination with pathologic data obtained from muscle biopsy. Even these studies resulted in nonspecific diagnosis until the advance of molecular genetic diagnosis. As new era of genetic analysis offers therapeutic options of experimental genetically modifiable drugs and clinical trials, this emphasizes appropriate diagnostic approach in this patient population. From this context, the authors discuss characteristics of pediatric neuromuscular disorders and existing diagnostic approaches in conjunction with new genetic diagnostic techniques for easier and less invasive diagnosis in these patients.

\section{Pediatric Neuromuscular Disorders}

Pediatric neuromuscular disorders are a group of disorders manifesting hypotonia, muscle weakness and hyporeflexia, originating from abnormality in peripheral nervous system and skeletal muscle. In particular, the lesions are spread throughout spinal anterior horn cell, peripheral nerves, neuromuscular junction and muscular structures [1-5]. When there is abnormality in these regions, the patients suffer chronic progressive or nonprogressive complications resulting from abnormal muscle function. Skeletal muscle weakness may lead to developmental delay in achieving gross motor milestone, gait abnormality, and sometimes even to loss of independent ambulation. Depends on the organs involved, the patients suffer respiratory failure from

Received: 24 May 2018, Revised: 10 November 2018, Accepted: 12 November 2018, Published: 31 December 2018

*Corresponding author: Young-Mock Lee, M.D., Ph.D. (iD http://orcid.org/0000-0002-5838-249X

Department of Pediatrics, Gangnam Severance Hospital, Yonsei University College of Medicine, 211 Eonju-ro, Gangnam-gu, Seoul 06273, Korea.

Tel: +82-2-2019-3354, Fax: +82-2-3461-9473, E-mail:ymleemd@yuhs.ac

Conflict of interest: The authors declare that they do not have any conflicts of interest.

(c) This is an open-access article distributed under the terms of the Creative Commons Attribution Non-Commercial License (http://creativecommons.org/licenses/by-nc/4.0/) which permits unrestricted non-commercial use, distribution, and reproduction in any medium, provided the original work is properly cited.

(c) Copyright 2018 by the Korean Society of Medical Genetics and Genomics 
respiratory muscle weakness, cardiomyopathy, arrhythmia and cardiac failure. Additionally, patients also can experience exercise intolerance, myalgia and rhabdomyolysis $[2,6]$.

\section{Importance of Establishing the Right Diagnosis}

Establishing the right diagnosis is important to predict prognosis for the patient, and to enable anticipatory care management for possible morbidity and multiorgan involvement [1-6]. Previously, most of the pediatric neuromuscular disorders were considered to be not curable and therefore the treatment goal was focused on supportive care to decrease mortality and morbidity. However, with huge improvement of molecular genetic diagnostic methods and resultant development of genetically modifiable drugs, some of the patients are now offered with therapeutic options once their diagnosis is confirmed, especially with genetic testing. In addition, accurate genetic diagnosis would enable better genetic counseling for the patients themselves and their families and relatives [5-8].

\section{Difficulty in Diagnostic Approach}

Nevertheless, it is hard to say that the genetic testing methods provide a one-and-only ultimate solution in diagnosing these children $[5,7,8]$. Accurate diagnosis requires complicated stepwise process starting from categorization according to clinical symptoms and signs, followed by several studies including laboratory tests, electrophysiologic studies such and EMG and NCS, pathology and immunohistochemistry data from muscle biopsy, and imaging studies like ultrasound and magnetic resonance imaging (MRI). Careful consideration of these information should be given prior to genetic testing, especially due to cost and difficulty in interpretation of the study result [1-5]. We will discuss further later in the manuscript regarding pros and cons of genetic testing method. Even though there are certain disease groups in this population which have relatively notable clinical features such as Duchenne muscular dystrophy (DMD) [9], spinal muscular atrophy (SMA) [10], and Facio-scapulohumeral muscular dystrophy (FSHD) [1,2], most other disease groups generally show clinical heterogeneity which complicates clinical categorization [5-8]. The clinical symptoms and signs are not specific for each diagnosis, and phenotypically overlapping. The studies currently used also complicates the diagnostic approach, as the results obtained from the laboratory tests, electrophysiologic study muscle biopsy data sometimes provide non-specific results, and do not indicate one particular diagno- sis [11-14]. In addition to that, it is hard to perform these studies in the pediatric population due to incooperation, necessitating sedation with anesthesia, which will be further discussed later. Even genetic testing and interpretation of the results are confusing that one genetic mutation may cause different diagnosis with overlapping clinical spectrum, while one clinical spectrum may be caused by multiple genetic mutations $[5,7,8]$. Moreover, even when variants of unknown significance are found with genetic testing, the road to the accurate diagnosis gets even more complicated. For example, in patients with nemaline myopathy, one subtype of congenital myopathies, there are 11 genetic loci known to be associated with the disease $[1,15,16]$. In contrast, when there is a mutation on the ryanodine receptor (RYR 1 ) gene, different pathologic features and variable clinical severity can be seen with this one gene mutation [17].

\section{Phenotype-down Diagnostic Approach}

\section{History, clinical examination and laboratory studies}

To reach an accurate diagnosis, "phenotype-down" approach has been widely used [5]. This approach involves stepwise application of diagnostic studies. Once again, as there are certain disease groups with characteristic clinical features, it is very important for the clinician to make a first step of categorization with detailed clinical examination and history taking. Information including prenatal and antenatal history, family history, timeline of motor milestone achievement and age of symptom onset needs to be obtained. General and neurological examinations including inspection of facial and body dysmorphism, skin abnormalities and organomegaly should also be performed. Integration of these information leads to prioritization of differential diagnoses $[1,2,4,7]$. As a next step, serum creatine kinase (CK) and aldolase will be obtained. Serum CK level gives clue in the differential diagnosis and elevated serum CK usually indicates an underlying neuromuscular condition, especially primary muscle disorders, according the severity of elevation. However, serum CK can still be mildly elevated in neurogenic disorders as well. If serum CK is considerably increased up to 5-10 times compared to reference value, the clinician may suspect the diagnosis of DMD and Becker muscular dystrophy (BMD) spectrum [9], as well as limbgirdle muscular dystrophy and congenital muscular dystrophy, which needs further evaluation $[18,19]$. In addition, in some cases when metabolic myopathies are suspected by history and clinical examination, the clinician can order metabolic workup including serum and urine studies. In metabolic myopathies, CK may be normal at rest and elevated after exercise, however, in 
McArdle disease, serum CK is usually elevated. Moreover, serum lactate and serum lactate to pyruvate ratio is usually elevated in mitochondrial disorders.

\section{Electrophysiologic studies (electromyography and nerve conduction study)}

The next step will be either electrophysiologic studies including EMG and NCS or pathologic study via muscle biopsy. Clinician should pay careful consideration when obtaining muscle biopsy as it is rather invasive in nature $[13,14]$ requiring sedation by anesthesia in most cases in the pediatric setting compared to serum studies and genetic testing available via simple blood draw. This is also applicable in some cases when perfoming needle EMG and NCS in very young children. In addition to the poor tolerability and difficulty in cooperation, technical difficulties and interpretational challenges from lack of experienced pediatric electrophysiologist hinders appropriate use of these studies in differential diagnosis. Yet, EMG and NCS can provide valuable information to differentiate between neuropathy and myopathy [11,12], which is very helpful, especially with older children with ability to cooperate and for the patients with neuromuscular junctional problems. In myopathies, EMG shows low amplitude, polyphasic and short duration potentials, and in neurogenic disorders, NCS shows fibrillation potentials, positive sharp waves, fasciculation potentials and signs of reinnervation. In myotonic disorders, EMG shows characteristic features of waxing and wane myotonic discharges with particular sound. Yet, EMG and NCS are not easy to obtain in neonates and infants and not very helpful in differentiating neuropathy and myopathy. This is because of need for sedation in very young children, and also because the reference normative values change over aging in these children, which requires expertise of the performing clinician [11-13].

In general, with the evolution of molecular genetic diagnosis, the need for electrophysiologic studies is decreasing [7]. Still, EMG and NCS will be helpful when the clinician cannot differentiate further to directly go into genetic testing, such as in motor unit disorders including SMA, hereditary motor and sensory neuropathies, Guillain-Barre syndrome, chronic inflammatory demyelinating polyneuropathy and disorders of the neuromuscular junction $[7,11]$.

\section{Muscle biopsy and pathologic studies}

Muscle biopsy can be obtained if the clinician suspects the diagnosis of muscular dystrophy or myopathy from the information obtained as above. Information regarding muscle histol- ogy, histochemistry, immunohistochemistry and ultrastructure can be obtained. The procedure of muscle biopsy is even more invasive compared to the previous studies that children mostly needs general anesthesia. If the clinician suspects a certain diagnosis with easier and less invasive genetic testing, muscle biopsy may not be necessary, such as in DMD, SMA, and FSHD. However, besides these particular disease group, muscle biopsy still provides significant information and is essential in diagnosis [7].

By obtaining histology and histochemistry data, information as below can be assessed: fiber size and shape, fiber type pattern, position of nuclei in muscle cell, degeneration, regeneration, fibrosis and fatty change, cellular reaction, structural change, vacuole, enzyme deficiency, glycogen or lipid accumulation. In neurogenic condition, the muscle biopsy shows small, angulated atrophied muscle fiber representing denervation, and fiber type grouping representing reinnervation. In myopathy, histology shows variety of structural defects and some are characteristic in certain disease groups, and histochemistry shows particular enzyme defect $[20,21]$. In muscular dystrophy, palestained or hypercontracted intensely stained degenerating fibers and regenerating fibers are seen, and necrosis and fibrosis are hallmarks of dystrophic muscles. By immunohistochemistry methods, one can assess absence, reduction or accumulation of certain proteins by labeling antibodies to specific proteins, which will be useful to reach a diagnosis, for example absence of laminin $\alpha 2$ suggests underlying merosin-deficient congenital muscular dystrophy [22]. With electron microscopy, detailed examination of ultrastructure and organelle of the muscle fiber and identification of the features seen with light microscopy such as vacuoles, rods, abnormal mitochondria or tubular aggregates $[1,7,14]$. These information can give clues to reach the diagnosis, yet not enough to confirm genetic change, especially regarding genetic and pathology overlap and heterogeneity in these disorders, again, necessitating further process of genetic mutation testing by panel $[1,2,5,7,8,15]$.

\section{Muscle and brain imaging: ultrasound and MRI}

Recently, muscle imaging including ultrasound and MRI is more widely used to differentiate different forms of congenital myopathy by evaluating selective muscle involvement pattern. Previously, computed tomography scan of the muscles have also been utilized; however, MRI has become the gold standard currently. Ultrasound has the advantage of easy utility in clinics not requiring sedation. MRI of the muscles not only shows extent of fibrofatty changes within the muscle compartment but also 
shows specific patterns of involvement in the muscle groups, like in BMD and RYR1-related myopathy. The imaging studies are also used to choose appropriate muscle to proceed with biopsy. However, the information from the imaging studies are variable for specificity and the interpretation is dependent on the expertise of the performing clinician and reading radiologist, and imaging study data should be considered in combination with other study data results including laboratory, electrophysiology and pathology studies [1-3].

Brain MRI scan also shows significant pattern in some of the neuromuscular conditions in children. For example, merosindeficient congenital muscular dystrophy shows significant white matter signal changes, and in Fukuyama type of congenital muscular dystrophy and muscle-eye-brain disease, brain MRI shows occipital pachygyria. In Walker-Warburg syndrome, brain MRI shows severe lissencephaly. In mitochondrial disorders, unilateral or bilateral signal change in basal ganglia is quite significant in Leigh disease, and in mitochondrial encephalomyopathy, lactic acidosis, and stroke-like episodes syndrome, acute focal lesions which occur in relation to stroke-like episodes, but occur outside the usual territories of vascular infarction are common [1].

\section{New Era of Molecular Genetic Testing and Inte- grated Diagnostic Approach}

There has been a huge improvement in the technique of molecular genetic diagnosis in this field for the past 15 years [7]. Techniques including multiplex ligation-dependent probe amplification (MLPA) and Sanger sequencing for specific genes are available, as well as further advanced targeted gene panels, targeted next generation sequencing (NGS) or whole exome sequencing (WES) methods. Obtaining genetic testing has advantages in pediatric population compared to other studies that they are less invasive and very accurate in achieving final diagnosis, mode of inheritance and risk of recurrence. By achieving the genetic diagnosis, it is also helpful for appropriate clinical management to decrease morbidity and mortality, better quality of life and application of therapeutic options of genetically modifiable drugs and clinical trials $[6-8,15]$.

In disease groups like SMA and DMD with notable clinical presentation and history, MLPA method to detect exon deletion or duplication is the first-tier test. If MLPA is negative in DMD, then Sanger sequencing of the dystrophin gene will be the second-tier test, and for other disease groups with particularly well-defined clinical phenotype, Sanger sequencing is the cost- effective, less time-consuming way to detect small sequence variation with higher diagnostic yield $[4,7,9]$. However, Sanger sequencing can analyze only one gene at a time. As we have mentioned earlier, most of other neuromuscular conditions in childhood share overlapping clinical features originating either from one gene or several different genes. Considering this point, targeted gene panels, targeted NGS or WES methods to screen hundreds to thousands of genes with a single reaction is widely used these days $[7-9,15]$.

Targeted gene panels and NGS methods have advantages of fast and increased throughput and coverage of regions of interest. In particular for neuromuscular conditions, these methods can also cover genes associated with metabolic conditions as well as mitochondrial disorders which significantly overlap clinical phenotype of myopathic features. However, they should be obtained with caution and should not be used as a first tier diagnostic test before comprehensive clinical evaluation and consideration of other supportive diagnostic methods. There are several challenges in interpreting the result from the NGS or the gene panel for the following reasons: firstly, clinician have to keep in mind that not all neuromuscular conditions in children are genetic diseases; secondly, there are weakness of either too much information vs. insufficient or unreliable information when interpreting the results, and this happens due to analysis of hundreds to thousands of genes with a single test. Sometimes, clinical may be provided with too much information of novel variant of unknown significance or previously unreported significance requiring futher functional validations. Or sometimes the results show too many polymorphisms not meeting clinial phenotypes which needs prioritization. On the other hand, sometime the results show insufficient or unreliable information regarding repeat expansions, copy number variation, and some giant genes to identify a pathogenic varient. In summary, despite the advantages of relatively rapid and cost-effectivity in screening multiple genes, clinicians should put effort in choosing the right panel and interpret the results with these methods. So far, it is reported that the diagnostic yield varies from 18-60\% depending on the disease subtype per literature [7-9,15].

For cost-effective, rapid and accurate diagnosis sparing timeconsuming and invasive diagnostic tests, early referral to pediatric neurologist or genetic specialist is very important. To achieve early referral, early detection and suspicion of neuromuscular disorders by clinician would be necessary. Considering that all infants and toddlers are to be seen by doctors on a regular basis for the national early childhood health examination under unified national health insurance system [23], screening of 
developmental milestones should better be done by certified pediatricians for this purpose. In addition, for floppy infants found in neonatal intensive care unit in their neonatal period, it is important that the neonatologist suspect and consult to pediatric neurologist or genetic specialist as early as possible [24-26].

In summary, when a child comes to see a pediatric neurologist or genetic specialist with suspicion of neuromuscular disorders, comprehensive diagnostic approach including detailed history taking, thorough clinical examination of the phenotype and previously used studies in combination with appropriate genetic testing should be done, in the most effective manner.

\section{Clinical Phenotype and Diagnostic Evaluations in the Most Common Forms of Pediatric Neuro- muscular Disorders}

Table 1 summarizes some of the most representative disease groups in this population and associated diagnostic test results. Detailed information for each disease group will be followed, and these disease groups favor certain priority in the diagnostic approach.

\section{Spinal muscular atrophy}

SMA arises from same genetic defect on chromosome $5 q 11$ 13, mutations in the survival motor neuron 1 (SMN1) gene throughout the clinical spectrum of SMA0-4. Additional testing of SMN2 copy number is usually obtained on a research basis, roughly reflecting better motor function with larger number of SMN2 copies. The clinical spectrum divides by the age of onset and maximal achievable motor function, from SMAO being the worst to SMA4 most mild adult form. Child neurologist usually see children from SMA1-3, and most of the children come to the clinical with hypotonia, weakness or delayed motor development, sometimes regressing motor milestone after infection. The patients have depressed or absent spinal reflex and sometimes show tongue fasciculation and hand tremor. With disease progression, they slowly weaken and develop thoracolumbar scoliosis and may need surgical correction, and respiratory issues might also develop necessitating ventilator support. Serum CK is usually mild to moderately elevated (less than 1,000 IU/L) but can be in normal range. With easily available genetic testing of MLPA to detect deletion of exons 7 and 8 of the SMN gene, EMG/NCS are less performed for the diagnosis of these children. However, EMG/NCS shows neuropathic features and may support and help earlier diagnosis in very young infant awaiting genetic testing result. Muscle biopsy is rare obtained in these children, and if obtained, will show neurogenic features of grouped atrophy $[2,4,10]$. Early confirmation of diagnosis in these children is especially crucial as there is newly Food and Drug Administration (FDA) approved genetic drugs such as Spinraza ${ }^{\circledR}$ (nusinersen; Biogen Inc., Cambridge, MA, USA) for SMA, as well as upcoming clinical trials using different genetic approach [27-30].

\section{Duchenne muscular dystrophy/Becker muscular dys- trophy}

DMD and BMD are clinical spectrum originating from the dystrophin gene mutation, DMD being the severe form and BMD being the milder form. Usually, DMD results from nonsense or frameshift mutation of dystrophin gene which fails to produce functional dystrophin protein, while BMD results from the socalled in-frame mutation which produce truncated partially functional dystrophin protein. DMD usually presents around age 3 to 5 years with gross motor developmental delay and Gowers' maneuver to stand up from the floor. Proximal lower extremity weakness is usually prominent from the presentation, and disease progression lead to loss of independent ambulation by age 8 to 12 years without intervention. Later in the disease course, patients will require ventilator assistant in their teens, and cardiac failure may follow. In comparison, BMD relatively presents later, generally asymptomatic during the infant and toddler ages and usually detected by incidental finding of elevated serum CK or positive family history when diagnosed during this early period. They present later than DMD and disease progress slower but in a similar manner. Serum CK is usually very high in DMD, at least 5 to 10 times elevated from normal values and sometimes up to hundreds from normal values. Typical clinical feature and history facilitates diagnostic decision and genetic testing is easily available. MLPA to detect exon deletion or duplication of dystrophin gene is used as a first step and if negative, Sanger sequencing to detect point mutation is available. With easy access of genetic testing, need for EMG/NCS and muscle biopsy are decreasing. If performed, EMG will show typical myopathic features yet it is unnecessary unless the clinical presentation is atypical and this is rare. Muscle biopsy might be necessary when MLPA is negative and the child has a positive family history and sequencing is not available, and the pathology will show dystrophic features of degenerating and regenerating fibers with fibrofatty replacement, and Western blot assay can quantitate dystrophin levels $[1,2,9]$.

Despite relatively fast disease progression, it is currently considered a standard of care to treat these patients with oral glu- 


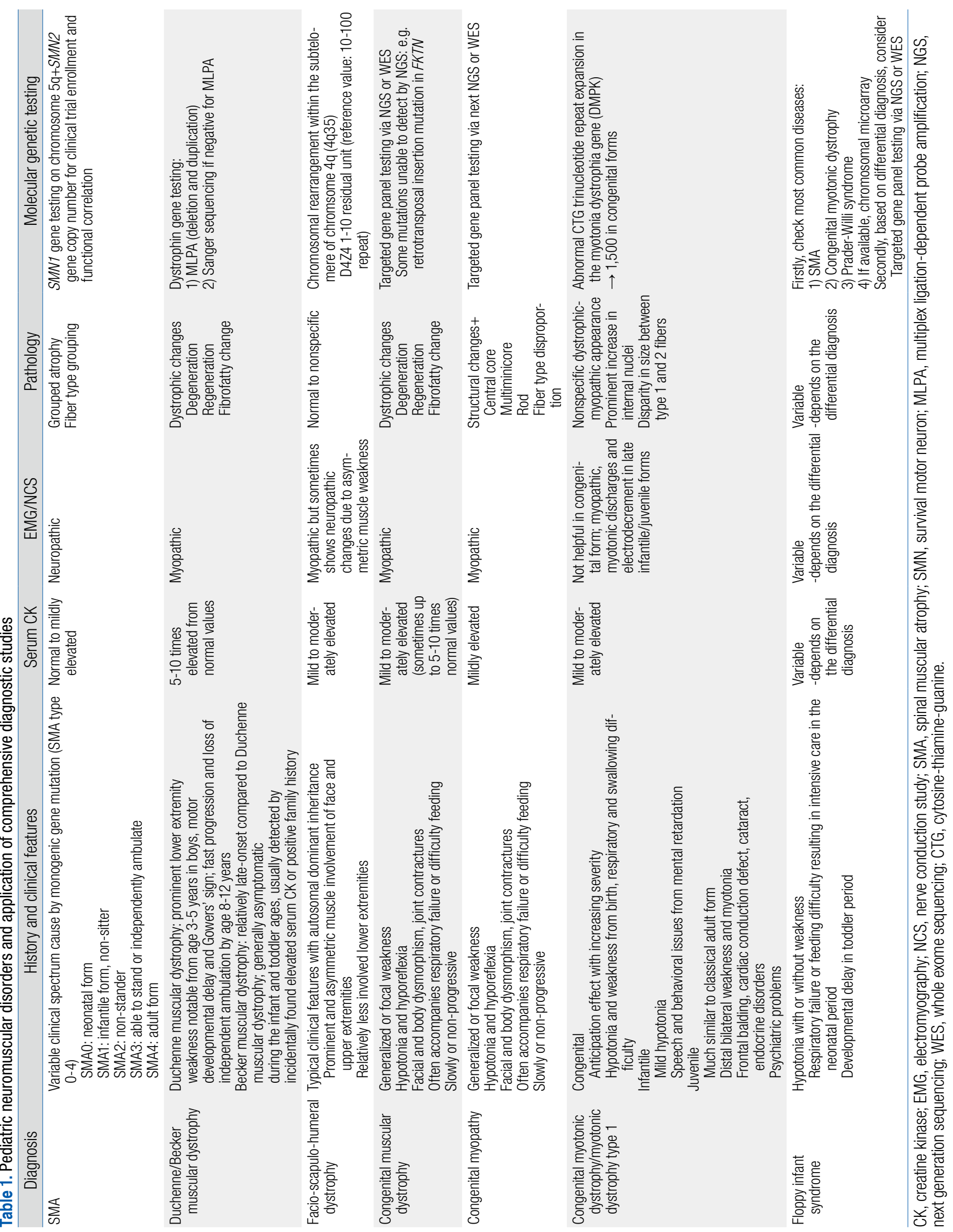


cocorticoids [31,32] as they delay disease progression, lengthen independent ambulation by preserving skeletal muscle and preserve respiratory muscle strength, decrease need for scoliosis surgery. In addition, there is a genetically modifiable drug recently approved by FDA, EXONDYS51 $1^{\mathrm{TM}}$ (eteplirsen; Sarepta Therapeutics, Cambridge, MA, USA) [33-36], as well as ongoing and upcoming clinical trials by different approach [37]. These therapeutic options make it even more important to diagnose these children accurate and right on time.

\section{Facio-scapulo-humeral muscular dystrophy}

FSHD also has a distinct clinical phenotype, and it is inherited in an autosomal dominant fashion. Prominent facial and scapula-humeral muscle weakness and asymmetric involvement is quite typical of these children, and genetic testing is easily available. DNA test shows an allele of decreased number of 1-10 residual $3.3 \mathrm{~kb}$ repeated DNA (D4Z4) units when in normal population, the repeat unit number varies from 10 to more than 100. EMG/NCS might be performed sometimes due to asymmetric muscle involvement which confuses clinician, and will show myopathic features of low amplitude, short duration motor unit potentials (MUP). Muscle biopsy in these children would show normal or nonspecific pathology, and generally not recommended for diagnosis $[1,2,4]$.

\section{Congenital myopathy and congenital muscular dystrophy}

These children usually presents at or slightly after birth with hypotonia, progressive or nonprogressive muscle weakness, some with respiratory and feeding problems and dysmorphic features. Clinical symptoms and signs are heterogenous and overlapping which complicates the diagnostic process. As a first step, serum CK will give a clue to differentiate between dystrophy and myopathy group that serum CK is usually much higher in dystrophy group, while in myopathy group serum CK is normal to mildly elevated. Even though targeted gene panel testing via NGS may look attractive as a shortcut, integrating other information including EMG/NCS, muscle biopsy and muscle and brain imaging would reach a final diagnosis avoiding confusion interpretating complex NGS results. In both dystrophy and myopathy groups, EMG will show normal or myopathic features of low amplitude compound muscle action potentials (CMAP) and small polyphasic recruitment of MUPs, as well as fibrillation potentials. Muscle biopsy will help differentiating myopathy and dystrophy group and lead to specific histologic diagnosis. Special structural change such as central nuclei, central core, multi- minicore, nemaline rod would be seen in myopathy group while degenerating and regenerating fibers and fibrofatty changes will be seen in dystrophy group. Immunohistochemistry studies will further necessitate diagnosis by revealing absence or accumulation of certain protein. Muscle ultrasound and MRI provide information regarding patterns of specific muscle involvement, and particular abnormality in brain MRI suggests disease such as Fukuyama type of congenital muscular dystrophy or WalkerWarburg syndrome. By combining these information, final diagnosis can be made and when obtaining and interpretating NGS results, clinician should keep in mind that there are certain disease groups and genetic mutations unable to detect by this method [1,2,5,20-22].

\section{Myotonic dystrophy type 1 and congenital myotonic dystrophy}

While myotonic dystrophy 1 (DM1) is one of the most common forms of muscular dystrophy in adult population, it is less common in children, and classified into congenital, infantile/ childhood and juvenile forms based on the age of onset and severity, and the clinical symptoms and signs are somewhat different from the classical adult DM1. However, DM1 and congenital DM shares genetic background the abnormal cytosinethiamine-guanine (CTG) trinucleotide repeat expansion in the myotonia dystrophia gene (DMPK), inherited in an autosomal dominant fashion. Classically, adult patients with DM1 shows clinical pattern of myotonia, muscular dystrophy, frontal balding, cataracts, cardiac conduction defects and endocrine disorders.

Clinical symptoms and signs are somewhat different in the congenital DM patients that they usually present with severe hypotonia and muscle weakness at birth, frequently accompanied by respiratory and feeding difficulties, followed by intellectual disability, autistic features, and abnormal musculoskeletal function, in general exhibiting more severe health issues. These babies commonly gets medical attention as floppy infants, and usually have previously known family history of DM or evidence of clinical myotonia in parental examination leads to the diagnosis by genetic testing. It is almost always maternally inherited. Anticipation is expected in these babies that with lower age of onset and successive generations, usually CTG repeat expansion is larger $(>1,500)$ and clinical severity increases. Yet in these babies, myotonia is not a prominent feature and not can be identified by the EMG during this period. When genetic testing is negative, muscle biopsy is necessary.

Clinically the infantile and juvenile forms are more similar to 
the adult form, yet the diagnosis is usually made later especially in the infantile form. Inheritance is maternal or paternal, and when younger, patients only exhibit mild hypotonia, lacking sucking and swallowing issues, nor dysmorphic features. They usually visit clinic with speech and learning difficulties and mental retardation which delays and complicates the diagnostic process. In these patients, the diagnosis process usually takes a step by step approach. In juvenile patients, clinical phenotype is much more similar to classical adult form with mild distal weakness and delayed muscle contraction, and accompanies other features aforementioned in the adult form. In these patients, serum CK is moderately high, and NCS is normal or shows decreased distal CMAPs, and EMG shows myopathic features with typical myotonic discharges, and repetitive nerve stimulation test reveals decrement at $10 \mathrm{~Hz}$, which leads to genetic testing of DMPK gene $[1,2,11,38]$.

\section{Floppy infants}

When it comes to the floppy neonates and infants, the diagnostic process is even trickier and time consuming, as being floppy not only contains group of patients with neuromuscular disorders but also patients with central nervous system anomalies or hypoxic brain damage and other metabolic conditions. So the diagnostic process would be performed step by step in a more conventional way. However, if the clinician suspects the neonates and infants to have neuromuscular condition, easy and less invasive genetic testing could be done. The testing should include SMA, congenital DM and Prader-Willi syndrome with one blood draw. Additional chromosomal microarray, if available, would be helpful in differentiating some of the most severe cases with relatively grave prognosis [24-26].

\section{Conclusion}

Diverse and nonspecific clinical symptoms and signs as well as overlapping genetic heterogeneity characterize pediatric neuromuscular disorders. For accurate and earlier diagnosis, conventional diagnostic methods of laboratory, electrophysiologic and pathologic studies will be helpful in combination with newer techniques of molecular genetic testing. Accurate genetic diagnosis in these patients will enable better clinical management and therapeutic options of clinical trials and new genetically modifiable drug treatment.

\section{References}

1. Goebel HH, Sewry CA, Weller RO. Muscle disease: pathology and genetics. 2nd ed. Chichester: Wiley-Blackwell; 2013.

2. Darras BT, Jones HR Jr, Ryan MM, De Vivo DC. Neuromuscular disorders of infancy, childhood, and adeloscence: a clinician's approach. 2nd ed. San Diego: Academic Press; 2014.

3. Piña-Garza JE, Fenichel GM. Fenichel's clinical pediatric neurology: a signs and symptoms approach. 7th ed. London: Elsevier Saunders; 2013.

4. Dubowitz V. Muscle disorders in childhood. 2nd ed. London: Bailliere Tindall; 1995.

5. North $\mathrm{KN}$, Wang $\mathrm{CH}$, Clarke $\mathrm{N}$, Jungbluth $\mathrm{H}$, Vainzof $\mathrm{M}$, Dowing JJ, et al. Approach to the diagnosis of congenital myopathies. Neuromuscl Dis 2014;24:97-116.

6. Dowling JJ, Gonorazky HD, Cohn RD, Campbell C. Treating pediatric neuromuscular disorders: the future is now. Am J Med Genet A 2018; 176:804-41.

7. Darras $B T$, Jones HR. Diagnosis of pediatric neuromuscular disorders in the era of DNA analysis. Pediatr Neurol 2000;23:289-300.

8. Ravenscroft G, Davis MR, Lamont $P$, Forrest $A$, Laing NG. New era in genetics of early-onset muscle disease: breakthroughs and challenges. Semin Cell Dev Biol 2017;64:160-70.

9. Emery AE, Muntoni F, Quinlivan R. Duchenne muscular dystrophy. 4th ed. Oxford: Oxford University Press; 2015.

10. Sumner CJ, Paushkin S, Ko CP. Spinal muscular atrophy: disease mechanisms and therapy. San Diego: Academic Press; 2016.

11. Preston DC, Shapiro BE. Electromyography and neuromuscular disorders: clinical-electrophysiologic correlations. 3rd ed. Oxford: Sauders; 2012.

12. Karakis I, Liew W, Darras BT, Jones HR, Kang PB. Referral and diagnostic trends in pediatric electromyography in the molecular era. Muscle Nerve 2014;50:244-9.

13. Rabie M, Jossiphov J, Nevo Y. Electromyography (EMG) accuracy compared to muscle biopsy in childhood. J Child Neurol 2007;22:8038.

14. Dubowitz V, Sewry CA, Oldfors A, Lane RJM. Muscle biopsy: a practical approach. 4th ed. Philadelphia: Saunders Elsevier; 2013.

15. Chae JH, Vasta V, Cho A, Lim BC, Zhang Q, Eun SH, et al. Utility of next generation sequencing in genetic diagnosis of early onset neuromuscular disorders. J Med Genet 2015;32:208-16.

16. Lim BC, Ki CS, Kim JW, Cho A, Kim MJ, Hwang H, et al. Fukutin mutations in congenital muscular dystrophies with defective glycosylation of dystroglycan in Korea. Neuromuscul Disord 2010;20:524-30.

17. Zvaritch E, Kraeva N, Bobardier E, McCloy RA, Depreux F, Holmyard D, et al. Ca2+ dysregulation in Ryr1 (I4895T/wt) mice causes congenital 
myopathy with progressive formation of minicores, cores, and nemaline rods. Proc Natl Acad Sci U S A 2009;106:21813-8.

18. Al-Ghamdi F, Darras BT, Ghosh PS. Spectrum of neuromuscular disorders with hyperCKemia from a tertiary care pediatric neuromuscular center. J Child Neurol 2018;33:389-96.

19. Kley RA, Shimidt-Wilcke T, Vorgerd M. Differential diagnosis of hyperCKemia. Neurol Intern Open 2018;2:E72-83.

20. North KN. Clinical approach to the diagnosis of congenital myopathies. Semin Pediatr Neurol 2011;18:216-20.

21. Böhm J, Vasli N, Malfatti E, Le Gras S, Feger C, Jost B, et al. An integrated diagnosis strategy for congenital myopathies. PLoS One 2013; 8:e67527.

22. Bönnemann $\mathrm{CG}$, Wang $\mathrm{CH}$, Quijano-Roy $\mathrm{S}$, Deconinck $\mathrm{N}$, Bertini $\mathrm{E}$, Ferreiro $A_{1}$ et al. Diagnostic approach to the congenital muscular dystrophies. Neuromuscl Disord 2014;24:289-311.

23. Eun BL, Kim SW, Kim YK, Kim JW, Moon JS, Park SK, et al. Introduction of national health screening program for infant and children. J Korean Child Neurol Soc 2007;15:142-7.

24. Sul YA, Yum MS, Lee YJ, Kim EH, Ko TS, Yoo HW. Floppy infant syndrome: clinical analysis and diagnositc approaches (2008-2012). J Korean Child Neurol Soc 2014;22:143-8.

25. Richer LP, Shevell MI, Miller SP. Diagnostic profile of neonatal hypotonia: an 11-year study. Pediatr Neurol 2001;25:32-7.

26. Paro-Panjan D, Neubauer D. Congenital hypotonia: is there an algorithm? J Child Neurol 2004;19:439-42.

27. Talbot K, Tizzano EF. The clinical landscape for SMA in a new therapeutic era. Gene Ther 2017;24:529-33.

28. Chiriboga CA, Swoboda KJ, Darras BT, lannaccone ST, Montes J, De Vivo DC, et al. Results from a phase 1 study of nusinersen (ISISSMN(Rx)) in children with spinal muscular atrophy. Neurology 2016; 86:890-7.

29. Finkel RS, Chiriboga CA, Vajsar J, Day JW, Montes J, De Vivo DC, et al. Treatment of infantile-onset spinal muscular atrophy with nusinersen: a phase 2, open-label, dose-escalation study. Lancet 2016;388:
3017-26.

30. Mercuri E, Darras BT, Chiriboga CA, Day JW, Campbell C, Connolly AM, et al. Nusinersen versus sham control in later-onset spinal muscular atropphy. N Engl J Med 2018;378:625-35.

31. McDonald CM, Henricon EK, Abresch RT, Duong $T_{1}$ Joyce NC, Hu F, et al. Long-term effects of glucocorticoids on function, quality of life, and survival in patients with Duchenne muscular dystrophy: a prospective cohort study. Lancet 2018;391:451-61.

32. McDonald CM, Gordish-Dressman H, Henricson EK, Duong T, Joyce $N C$, Jhawar $S$, et al. Longitudinal pulmonary function testing outcome measures in Duchenne muscular dystrophy: long-term natural history with and without glucocorticoids. Neuromuscul Disord 2018; 28:897-909.

33. Mendell JR, Goemans N, Lowes LP, Alfano LN, Berry K, Shao J, et al. Longitudinal effect of eteplirsen versus historical control on ambulation in Duchenne muscular dystrophy. Ann Neurol 2016;79:257-71.

34. Charleston JS, Schenell FJ, Dworzak J, Donoqhue $C$, Lewis S, Chen L, et al. Eteplirsen treatment for Duchenne muscular dystrophy: exon skipping and dystrophin production. Neurology 2018;90:e2146-54.

35. Voit T, Topaloglu H, Straub V, Muntoni F, Deconinck N, Champion G, et al. Safety and efficacy of drisapersen for the treatment of Duchenne muscular dystrophy (DEMAND II): an exploratory, randomized, placebo-controlled phase 2 study. Lancet Neurol 2014;13:987-96.

36. Goemans NM, Tulinius $M$, van den Hauwe $M$, Kroksmark AK, Buyse $G$, Wilson RJ, et al. Long-term efficacy, safety, and pharmacokinetics of drisapersen in Duchenne muscular dystrophy: results from an openlabel extension study. PLoS One 2016;11:e0161955.

37. McDonald CM, Campbell C, Torricelli RE, Finkel RS, Flanigan KM, Goemans $N_{1}$ et al. Ataluren in patients with nonsense mutation Duchenne muscular dystrophy (ACT DMD): a multicentre, randomized, doubleblind, placebo-controlled, phase 3 trial. Lancet 2017;390:1489-98.

38. Ho G, Carey KA, Cardamone M, Farrar MA. Myotonic dystrophy type 1: clinical manifestations in children and adolescents. Arch Dis Child 2018, in press. 